\title{
Role of coagulase-negative staphylococci and micrococci in ocular disease
}

\author{
VM MAHAJAN, TA ALEXANDER, RK JAIN, AND LP AGARWAL \\ From the Dr Rajendra Prasad Centre for Ophthalmic Sciences, All India Institute of Medical Sciences, \\ Ansari Nagar, New Delhi 110029, India
}

SUMMARY Forty-nine strains of coagulase-negative staphylococci and 26 strains of micrococci isolated from human eyes were classified by the Baird-Parker system of 1966. The staphylococci belonged to subgroups II, III, IV, and V with one strain each in subgroups III and IV. However, when biotyped by Baird-Parker's system of 1974 , less than $50 \%(23 / 49)$ of strains were typable and they belonged to biotypes 1 and 2 . With our proposed modified classification all strains could be classified into biotypes 1, 2, and 3 . All the 26 strains of micrococci belonged to subgroup VII.

Staphylococci isolated from diverse sources produced, qualitatively and quantitatively, different corneal pathology in rabbits when compared to those isolated from healthy conjunctiva. This bore no relation to the present system of subtyping based on biochemical reactions. Micrococci were incapable of producing experimental corneal lesions in the rabbit.

Coagulase-negative staphylococci and micrococci are being increasingly accepted as human pathogens. ${ }^{1}$ In ocular infections, Staphylococcus epidermidis has been recognised as a pathogen in corneal ulcers ${ }^{2}$ and several postoperative infections including endophthalmitis. ${ }^{3-5}$ It has also been held to be responsible for cases of blepharoconjunctivitis, ${ }^{6}$ and it is known to be pathogenic for the eye of the rabbit. ${ }^{6} 7$ Similar information concerning micrococci is lacking. The purpose of this communication is to biotype and to establish the ocular pathogenicity in the rabbit of a collection of staphylococci and micrococci from the human eye.

\section{Material and methods}

Material was collected and processed as previously described. 8 Preoperative conjunctival swabs were collected from patients who had come mostly for cataract extraction. From corneal ulcer cases swabs were invariably collected from the ulcerated area. Postoperative infections included surgical wounds, endophthalmitis, and infections of corneal grafts, uvea, and socket.

Swabs were streaked immediately on sheep blood agar medium. Strains were classified according to Baird-Parker, ${ }^{9} 10$ carbohydrate reactions being observed on solid media in Petri dishes. Phosphatase

Received for publication 25 February 1980 production was determined by both the Petri-dish and tube methods. ${ }^{11}$ Acetoin production was tested using Barrit's method, and the sensitivity of these organisms to penicillin, tetracycline, chloramphenicol, streptomycin, erythromycin, framycetin, gentamicin, cloxacillin, and novobiocin was tested by the disc diffusion technique. The results were interpreted as recommended by Bauer et al. ${ }^{12}$

\section{EXPERIMENTAL STUDY}

Twenty rabbits, in four groups, were inoculated with strains of subgroup V staphylococci and micrococci. Group 1, consisting of six rabbits, were given intracorneal injections of Staph. epidermidis isolated from healthy conjunctival sacs. Three strains were tested, two eyes per strain, and the remaining six eyes were controls. Groups 2 and 3 also comprised six rabbits each. In group 2, three strains of Staph. epidermidis isolated from cases of corneal ulcer were tested, and in group 3, three strains isolated from postoperative endophthalmitis, suture abscess, and uveitis were used. In group 4, three strains of micrococci, one each from a normal sac, a corneal ulcer, and a case of endophthalmitis, were studied on three eyes of two rabbits. The fourth eye was kept as a control, sterile saline being injected intracorneally. Staphylococci and micrococci, suspended in saline containing approximately $5 \times 10^{12}$ and $11 \times 10^{12}$ viable organisms $/ \mathrm{ml}$, were injected in doses of 0.01-0.02 ml.

Ocular examination was carried out every 24 
Table 1 Correlation of clinical category with biotype of strains of Staphylococcus epidermidis isolated from 49 patients

\begin{tabular}{|c|c|c|c|c|c|c|c|c|c|c|c|c|}
\hline \multirow[t]{2}{*}{ Clinical category } & \multicolumn{4}{|c|}{ Baird-Parker ${ }^{9}$ subgroup } & \multicolumn{4}{|c|}{ Baird-Parker ${ }^{10}$ biotype } & \multicolumn{4}{|c|}{ Modified classification biotype } \\
\hline & $I I$ & $I I I$ & $I V$ & $\boldsymbol{V}$ & 1 & 2 & 3 & 4 & $l$ & 2 & 3 & 4 \\
\hline Corneal ulcer & 9 & 1 & - & 1 & 9 & 1 & - & - & 9 & 1 & 1 & - \\
\hline \multicolumn{13}{|l|}{ Postoperative infection } \\
\hline Endophthalmitis & 3 & 一 & - & 3 & 3 & 一 & 一 & - & 3 & 一 & 3 & - \\
\hline Postkeratoplasty & 1 & 一 & 1 & 4 & 1 & - & - & - & 1 & - & 5 & 一 \\
\hline Contracted socket & 1 & 一 & - & - & 1 & 一 & 一 & 一 & 1 & - & - & - \\
\hline Postcataract and glaucoma surgery & 2 & - & 一 & 8 & $2 *$ & - & - & - & 2 & - & 8 & - \\
\hline Total & 22 & 1 & 1 & 25 & $22^{*}$ & 1 & 一 & - & 22 & 1 & 26 & - \\
\hline
\end{tabular}

* Remaining 26 did not fit into classical biotypes.

hours, and $50 \%$ of the eyes were enucleated immediately after regression of the lesions. The corneae were removed aseptically. One half was cultured, and the other half was examined histologically after staining with haematoxylin and eosin.

\section{Results}

Of 49 strains of catalase-positive, coagulase-negative staphylococci, $25(51.0 \%)$ belonged to subgroup V, $22(44.9 \%)$ to subgroup II, and one strain each to subgroups III and IV of Baird-Parker. ${ }^{9}$ In corneal ulcers, subgroup II strains $(9 / 11)$ predominated, whereas in postoperative infections subgroup $\mathrm{V}$ strains $(16 / 24)$ were more numerous. Nearly $50 \%$ of strains could not be biotyped by the latest classification proposed by Baird-Parker. ${ }^{10}$ Most of our strains produced acid aerobically from lactose and maltose, but all the strains were classified into types 1,2 , and 3 if these two tests were ignored (Table 1). The proposed modified scheme for biotyping is as follows:

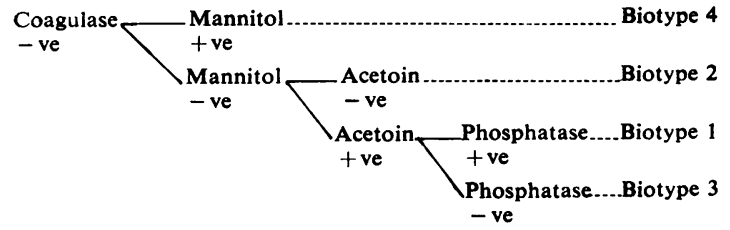

Correlation of clinical categories with different types of Staph. epidermidis is shown in Table 1. Most strains were sensitive to gentamicin (26) followed by framycetin (25), chloramphenicol (19), erythromycin (18), streptomycin (12), and tetracycline (8). Fewest strains (7) were sensitive to penicillin. The resistant strains were sensitive to cloxacillin. Antibiotic sensitivities did not appear to be related in any way to particular staphylococcal subgroups or to any clinical category. Of 29 strains tested for sensitivity to $30 \mu \mathrm{g}$ of novobiocin, five were resistant.

All 26 strains of micrococci belonged to type VII (Table 2). Most of them were sensitive to gentamicin, tetracycline, and erythromycin (25) followed by chloramphenicol (23), framycetin (19), and streptomycin (15). Only five were sensitive to penicillin. Of 25 strains tested for sensitivity to $30 \mu \mathrm{g}$ of novobiocin, only one strain was resistant. Sensitivity patterns were not related to clinical categories.

\section{EXPERIMENT AL ST UDY}

In group 1, all three strains produced superficial central corneal ulcers by the third day (Fig. 1), which healed with a leucomatous opacity in 15 days. In tissue sections, epithelium was deficient in the central part of the cornea, and only the anterior stroma was diffusely infiltrated (Fig. 2). In group 2, the pathology involved deeper layers of the cornea,

Table 2 Correlation of clinical category with biotype of strains of micrococci isolated from 26 patients

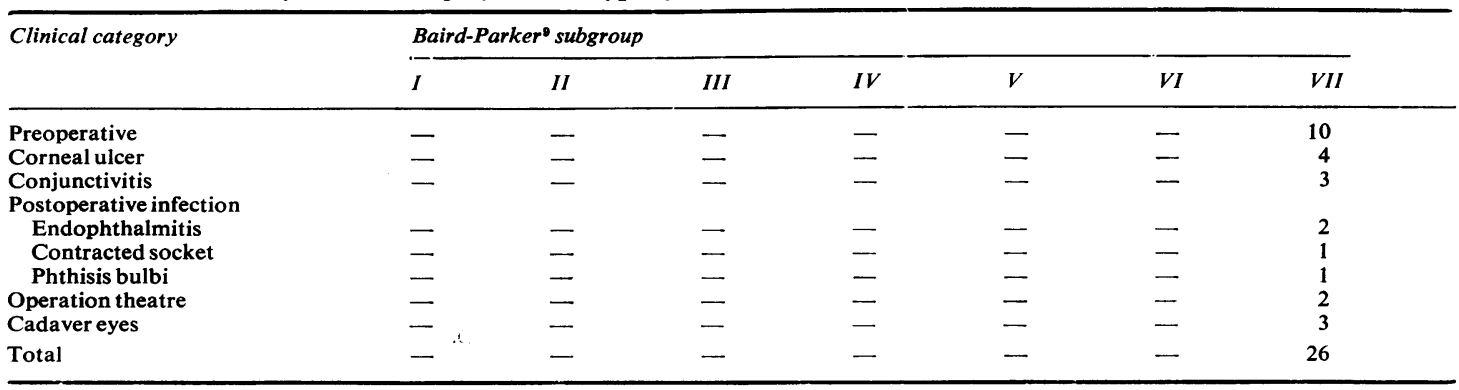




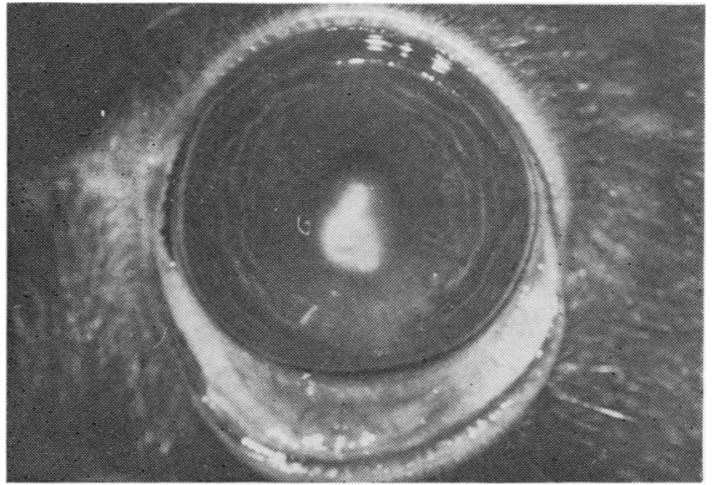

Fig. 1 Rabbit eye showing a small central corneal ulcer six days after intracorneal injection of Staph. epidermidis (group 1).

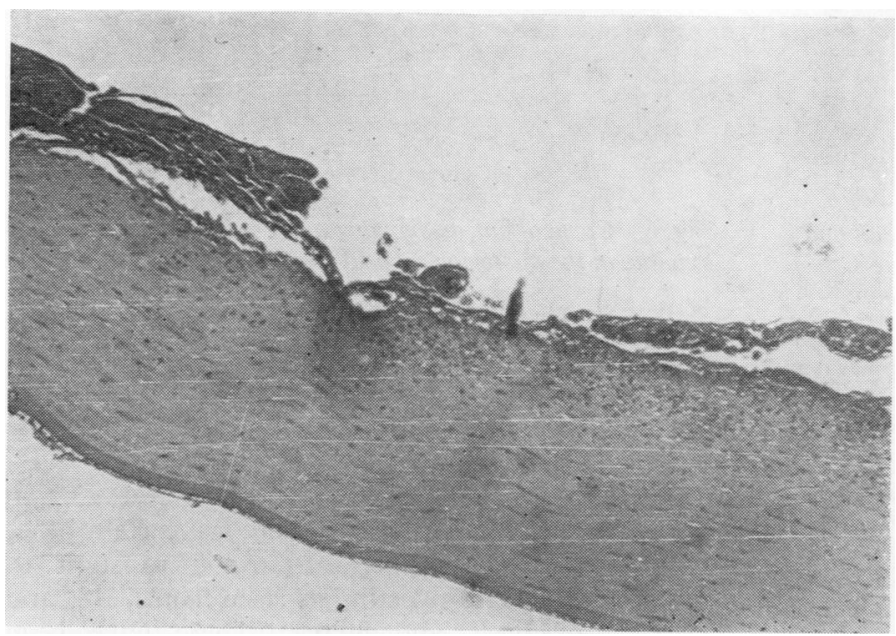

leading to intracorneal abscess formation with hypopyon (Fig. 3). The lesions healed in 27 days with vascularisation. Histopathology revealed involvement of the anterior and deeper layers of the cornea (Fig. 4). In group 3, the strains produced diffuse involvement of the cornea. Posterior corneal abscesses and profuse vascularisation were prominent features (Fig. 5). Histologically, the anterior layers were less involved than the posterior cornea. The retrocorneal region showed an acute inflammatory exudate (Fig. 6). Cultured corneal buttons yielded a pure growth of Staph. epidermidis of the same subtypes. In group 4 , the strains failed to produce any corneal pathology.

Control eyes became clear within 24 hours.
Fig. 2 Corneal button showing superficial ulceration, epithelium being deficient in the centre. Anterior stroma showing diffuse infiltration with acute inflammatory cells. Deeper layers are relatively unaffected. Haematoxylin and eosin $\times 80$.

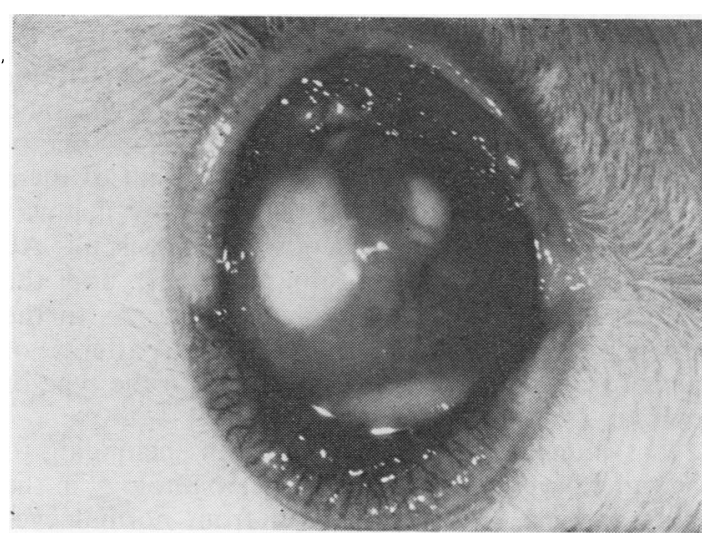

Fig. 3 Rabbit eye showing corneal ulcer with oedema, infiltration, and hypopyon nine days after injection of staphylococci (group 2).

\section{Discussion}

In the year 1976-77, of 88 strains of coagulasenegative staphylococci typed, 45 were subgroup II, 27 subgroup $\mathrm{V}$, and the remaining 16 subgroup IV. ${ }^{8}$ Of 49 such strains typed in the present study, subgroup $\mathrm{V}$ strains are more numerous than subgroup II. Similarly, postoperative infections (16) were mainly due to subgroup $\mathrm{V}$ compared with seven caused by subgroup II strains, whereas in the previous study postoperative infections were more commonly due to subgroup II strains. However, those isolated from corneal ulcers were subgroup II, in contrast to the previous finding of subgroup $\mathrm{V}$ strains. This is because the phosphatase test was done by the tube method ${ }^{11}$ in this study. With the Petri-dish method used in the earlier study, a sizeable number of strains produced doubtful results. In the present study also, the Petri-dish method produced 


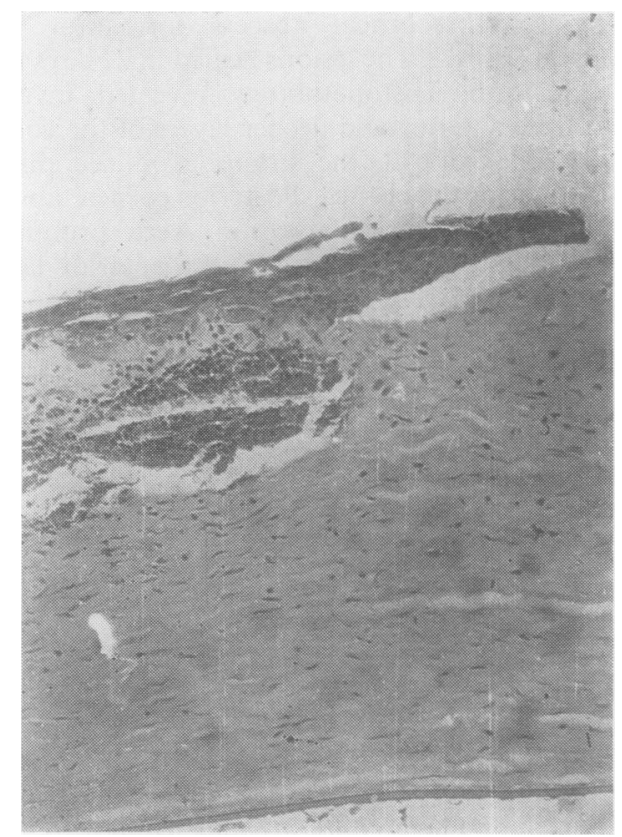

Fig. 4 Corneal button showing deficient epithelium and stroma in the central region. Infiltration with acute inflammatory cells is seen in anterior as well as deep stroma. $H$ and $E \times 80$.

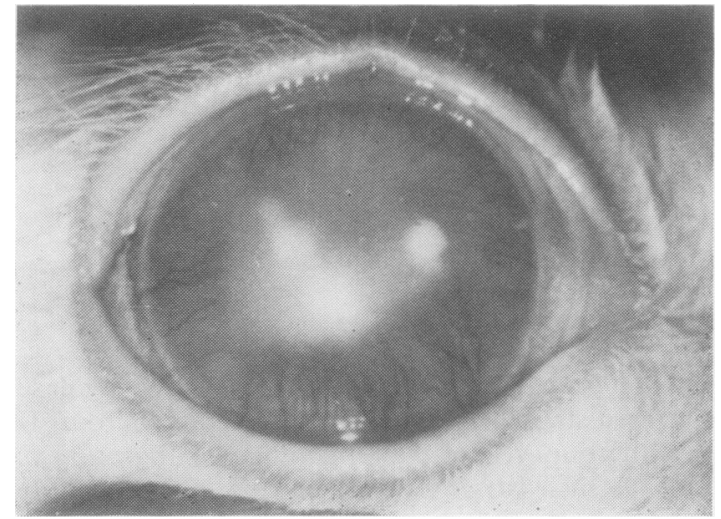

Fig. 5 Rabbit cornea showing intrastromal abscess and profuse vascularisation 15 days after injection of staphylococci (group 3).

false results in 10 strains $(20 \%)$ when verified by the tube method. The importance of this test was further appreciated when 26 strains, which could not be typed by the recent classification of Baird-Parker, ${ }^{10}$ were typable if acid production from lactose and maltose was ignored, as shown by the proposed classification. We, therefore, recommend that the phosphatase test should be done by the tube method

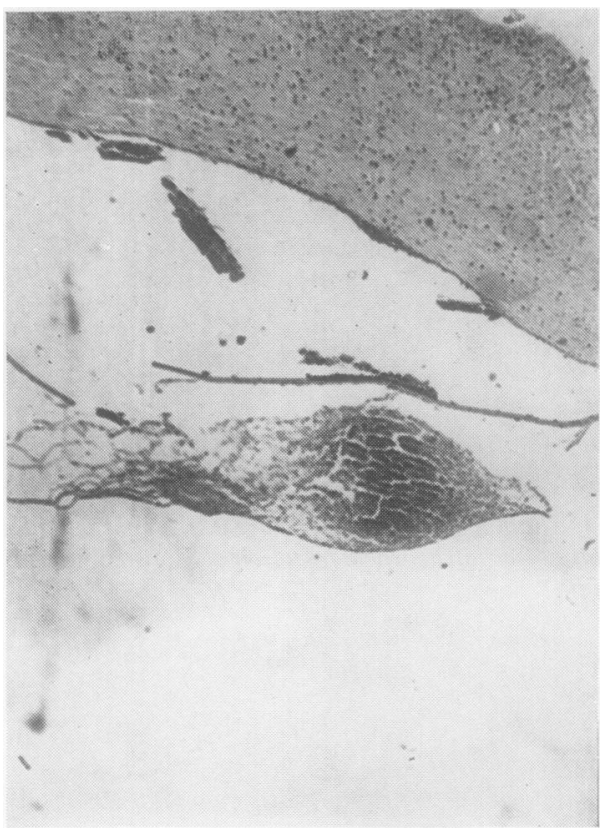

Fig. 6 Corneal button showing acute inflammatory exudate in the posterior corneal region. $H$ and $E \times 26$.

when classifying staphylococci or micrococci. The present study shows the importance of hospital strains of subgroup $V / 3$ biotype in postoperative infection though they may also be produced by the preoperative flora.

Resistance to penicillin has dropped from $92 \%$ in our previous study to $86 \%$. There are certain other differences. The number of strains resistant to gentamicin and framycetin has risen from $7.0 \%$ and $11.0 \%$ to $47.0 \%$ and $49.0 \%$. There have been marginal increases in resistance to other antibiotics (Table 3). Micrococci showed a similar pattern of resistance to penicillin $(80.8 \%)$, but only $4 \%$ strains were resistant to gentamicin, erythromycin, and tetracycline. Mitchell ${ }^{13}$ tested 30 strains of micrococci from the urinary tract and found all of them resistant to penicillin, while Corse and Williams ${ }^{14}$ reported $28 \%$ of their collection to be resistant. All our strains belonged to subgroup VII, and the source was the human conjunctival sac. As in the case of staphylococci, the resistance patterns of micrococci do not seem to be related to the type of lesion.

It is interesting to note that strains belonging to the same biotype produced varying pathology in the rabbit cornea. Those recovered from normal eyes produced minimal superficial epithelial changes. Those from corneal ulcers produced deeper involvement, and those from postoperative infections 
Table 3 Antibiotic resistance patterns (\%) of staphylococci and micrococci isolated from ocular tissues

\begin{tabular}{|c|c|c|}
\hline Antibiotic & Staphylococci & Micrococci \\
\hline Penicillin & $\begin{array}{c}85 \cdot 7 \\
(92 \cdot 0)\end{array}$ & $88 \cdot 8$ \\
\hline Chloramphenicol & $\begin{array}{c}61 \cdot 0 \\
(75 \cdot 0)\end{array}$ & $11 \cdot 5$ \\
\hline Gentamicin & $\begin{array}{l}47 \cdot 0 \\
(7 \cdot 0)\end{array}$ & $4 \cdot 0$ \\
\hline Framycetin & $\begin{array}{c}49 \cdot 0 \\
(11.0)\end{array}$ & $27 \cdot 0$ \\
\hline Erythromycin & $\begin{array}{c}63.3 \\
(59 \cdot 0)\end{array}$ & $4 \cdot 0$ \\
\hline Streptomycin & $\begin{array}{c}75 \cdot 5 \\
(62 \cdot 5)\end{array}$ & $42 \cdot 0$ \\
\hline Tetracycline & $\begin{array}{c}84 \cdot 0 \\
(83 \cdot 0)\end{array}$ & $4 \cdot 0$ \\
\hline
\end{tabular}

Figures in parentheses denote percentages in our previous study. ${ }^{8}$

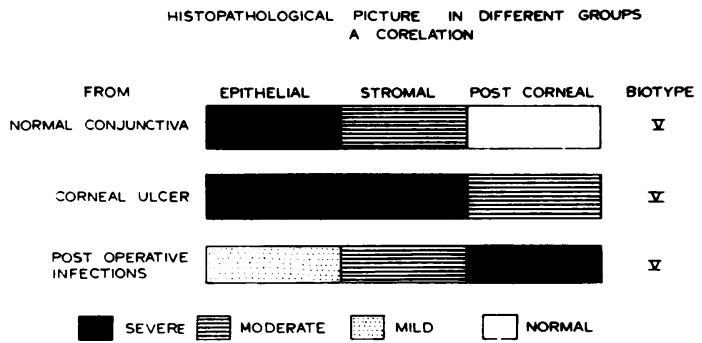

Fig. 7 A correlation of the histopathological picture obtained in the different groups.

produced diffuse involvement of the cornea in the form of posterior corneal abscess (Fig. 7). It would be interesting to see if strains of subgroup II/biotype 1 recovered from different conditions would show similar results. This is necessary before hypothesising that the present classification of staphylococci based on certain biochemical features is inadequate. Valenton and Okumoto ${ }^{6}$ reported that strains from subjects without disease were not dermonecrotoxic in rabbits, while those isolated from patients with disease were. The studies of our clinical colleagues have shown that corneal ulcers or postoperative infections due to Staph. epidermidis responded to the timely application of appropriate antibiotics. ${ }^{15}$ In a number of cases, visual acuity was restored.

Micrococci failed to produce disease in rabbits after the intracorneal injection of almost double the number of organisms that produced staphylococcal lesions.

\section{References}

${ }^{1}$ Meers PD, Whyte W, Sandys G. Coagulase negative staphylococci and micrococci in urinary tract infections. $J$ Clin Pathol 1975;28:270-3.

${ }^{2}$ Khosla PK, Prakash OM, Agarwal LP. Clinico-bacteriological study of soframycin in conjunctival flora (normal and corneal ulcer cases). Oriental Archives of Ophthalmology $1963 ; 1: 212-20$.

${ }^{3}$ Khosla PK, Angra SK, Agarwal LP. Post-operative staphylococcal infection. Oriental Archives of Ophthalmology $1964 ; 2: 240-2$.

4 Allen MF, Mangiaracine AB. Bacterial endophthalmitis after cataract extraction. Arch Ophthalmol 1964;72: 454-62.

${ }^{5}$ Valenton MJ, Brubaker RF, Allen HF. Staphylococcus epidermidis (albus) endophthalmitis (report of two cases after cataract extraction). Arch Ophthalmol 1973;89: 94-6.

- Valenton MJ, Okumoto M. Toxin producing strains of Staphylococcus epidermidis (albus). Arch Ophthalmol $1973 ; 89: 186-9$.

7 Agarwal LP, Nair RV, Sood NN, Mahajan VM. Role of trauma and corticosteroids in causation of corneal pathology by commensals. Eastern Archives of Ophthalmology 1976;4:1-6.

${ }^{8}$ Mahajan VM. Classification of staphylococci isolated from ocular tissues. J Clin Pathol 1979;32:396-8.

${ }^{9}$ Baird-Parker AC. Methods for classifying staphylococci and micrococci. In Identification Methods for Microbiologists Part A (Society for Applied Bacteriology Technical Series No. 1) ed BM Gibbs, FA Skinner. New York and London: Academic Press, 1966;59.

${ }^{10}$ Baird-Parker AC. The basis for the present classification of staphylococci and micrococci. Ann NY Acad Sci 1974; 236:7-13.

${ }^{11}$ Pennock CA, Huddy RB. Phosphatase reaction of coagulase negative staphylococci and micrococci. $J$ Pathol 1967;93:685-8.

${ }^{12}$ Bauer AW, Kirby WMM, Sherris JC, Turck M. Antibiotic susceptibility testing by a standardised single disk method. Am J Clin Pathol 1966;45:493-6.

${ }^{13}$ Mitchell RG. Classification of Staphylococcus albus strains isolated from the urinary tract. J Clin Pathol 1968;21: 93-6.

${ }^{14}$ Corse J, Williams REO. Antibiotic resistance of coagulase negative staphylococci and micrococci. J Clin Pathol $1968 ; 21: 722-8$.

15 Agarwal LP, Alexander TA, Mahajan VM, Ratnakar KS. Staphylococcus albus in ocular infections-a clinicobacteriological correlation and experimental study. Eastern Archives of Ophthalmology in press.

Requests for reprints to: Dr VM Mahajan, Dr Rajendra Prasad Centre for Ophthalmic Sciences, Ansari Nagar, New Delhi 110029, India. 